\title{
UNIFIED PRESENTATION OF CERTAIN FAMILIES OF ELLIPTIC-TYPE INTEGRALS RELATED TO EULER INTEGRALS AND GENERATING FUNCTIONS
}

\author{
V. B. L. CHAURASIA AND R. C. MEGHWAL
}

\begin{abstract}
The aim of the present paper is to give unified presentation of certain families of elliptic-type integrals related to Euler integrals and generating functions. It is a unification and generalization of certain families of elliptic-type integrals which were studied in a number of earlier works on the subject due to their importance and potential in certain problems in radiation physics and nuclear technology. The results established in this paper are of manifold generality and basic in nature. Besides deriving various known and new elliptic-type integrals and their generalizations these theorems can be used to evaluate various Euler-type integrals involving a number of generating functions.
\end{abstract}

\section{Introduction}

$$
\Omega_{j}(k)=\int_{0}^{\pi}\left(1-k^{2} \cos \theta\right)^{-j-\frac{1}{2}} d \theta,
$$

where $j=0,1,2, \ldots$ and $0 \leq k<1$ was studied by Epstein-Hubbel [5], for the first time. Due to its occurrence in a number of physical problems [1, 2, 6, 8, 15, 17, 29], in the form of single and multiple integrals, several authors notably Kalla [9, 10] and Kalla et al. [11], Kalla and Al-Saqabi [12], Kalla et al. [13], Salman [23], Saxena et al. [26], Srivastava and Bromberg [31], have investigated various interesting unifications (and generalizations) of the elliptic-type integrals (1). Some of the important generalizations of elliptic-type integrals (1) are as follows. Kalla $[9,10]$ introduced the generalization of the form:

$$
R_{\mu}(k, \alpha, \gamma)=\int_{0}^{\pi} \frac{\cos ^{2 \alpha-1}(\theta / 2) \sin ^{2 \gamma-2 \alpha-1}(\theta / 2)}{\left(1-k^{2} \cos \theta\right)^{\mu+\frac{1}{2}}} d \theta,
$$

where $0 \leq k<1, \operatorname{Re}(\gamma)>\operatorname{Re}(\alpha)>O, \operatorname{Re}(\mu)>-\frac{1}{2}$.

Results for this generalization are also derived by Glasser and Kalla [7]. Al-Saqabi [18] defined and studied the generalization given by the integral

$$
B_{\mu}(k, m, v)=\int_{0}^{\pi} \frac{\cos ^{2 m}(\theta) \sin ^{2 v}(\theta)}{\left(1-k^{2} \cos \theta\right)^{\mu+\frac{1}{2}}} d \theta,
$$

Corresponding author: V. B. L. Chaurasia.

2010 Mathematics Subject Classification. 33C65, 33E05, 33C70, 33C75.

Key words and phrases. Elliptic-type integrals, Euler-type integrals, generating functions, $\mathrm{H}$-function of several variables. 
where $0 \leq k<l, m \in N_{0}, \mu \in C, \operatorname{Re}(\mu)>-\frac{1}{2}$.

Asymptotic expansion of (3) has recently been discussed by Matera et al. [20]. The integral

$$
\Lambda_{\nu}(\alpha, k)=\int_{0}^{\pi} \frac{\exp \left[\alpha \sin ^{2}(\theta / 2)\right]}{\left(1-k^{2} \cos \theta\right)^{\nu+\frac{1}{2}}} d \theta,
$$

where $0 \leq k<1, \alpha, v \in R$; presents another generalization of (1), given by Siddiqi [29].

Srivastava and Siddiqi [35] have given an interesting unification and extension of the families of elliptic-type integrals in the following form:

$$
\Lambda_{(\lambda, \mu)}^{(\alpha, \beta)}(\rho ; k)=\int_{0}^{\pi} \frac{\cos ^{2 \alpha-1}(\theta / 2) \sin ^{2 \beta-1}(\theta / 2)}{\left(1-k^{2} \cos \theta\right)^{\mu+\frac{1}{2}}}\left[1-\rho \sin ^{2}(\theta / 2)\right]^{-\lambda} d \theta,
$$

where $0 \leq k<1, \operatorname{Re}(\alpha)>O, \operatorname{Re}(\beta)>0, \lambda, \mu \in C,|\rho|<1$.

Kalla and Tuan [14] generalized equation (5) by means of the following integral and also obtained its asymptotic expansion

$$
\Lambda_{(\lambda, \gamma, \mu)}^{(\alpha, \beta)}(\rho, \delta ; k)=\int_{0}^{\pi} \frac{\cos ^{2 \alpha-1}(\theta / 2) \sin ^{2 \beta-1}(\theta / 2)}{\left(1-k^{2} \cos \theta\right)^{\mu+\frac{1}{2}}}\left[1-\rho \sin ^{2}(\theta / 2)\right]^{-\lambda}\left[1+\delta \cos ^{2}(\theta / 2)\right] d \theta,
$$

where $0 \leq k<1, \operatorname{Re}(\alpha)>0, \operatorname{Re}(\beta)>0, \lambda, \mu, \gamma \in C$ and either $|\rho|,|\delta|<1$ or $p$ (or $S$ ) $\in C$ whenever $\lambda=m$ or $\gamma=-m, m \in N_{0}$, respectively.

Al-Zamel et al. [19] discussed a generalized family of elliptic-type integrals in the form:

$$
\begin{aligned}
Z_{(\gamma)}^{(\alpha, \beta)}(k) & =Z_{\left(\gamma_{1}, \ldots, \gamma_{n}\right)}^{(\alpha, \beta)}\left(k_{1}, \ldots, k_{n}\right) \\
& =\int_{0}^{\pi} \cos ^{2 \alpha-1}(\theta / 2) \sin ^{2 \beta-1}(\theta / 2) \prod_{j=1}^{n}\left(1-k_{j}^{2} \cos \theta\right)^{-\gamma_{j}} d \theta \\
& =B(\alpha, \beta) \prod_{j=1}^{n}\left(1-k_{j}^{2}\right)^{-\gamma_{j}} F_{D}^{(n)}\left(\beta ; \gamma_{1}, \ldots, \gamma_{n} ; \frac{2 k_{1}^{2}}{k_{1}^{2}-1}, \ldots, \frac{2 k_{n}^{2}}{k_{n}^{2}-1}\right),
\end{aligned}
$$

where $\operatorname{Re}(\alpha)>O, \operatorname{Re}(\beta)>O,\left|k_{j}\right|<1 ; \gamma_{j} \in C(j=1, \ldots, n) F_{D}^{(n)}(\cdot)$ is the Lauricella hypergeometric function of $n$-variables [21, p.163].

Saxena and Kalla [27] have studied a family of elliptic-type integrals of the form:

$$
\begin{aligned}
& \Omega_{\left(\sigma_{1}, \ldots, \sigma_{n-2} ; \delta, \mu\right)}^{(\alpha, \beta)}\left(\rho_{1}, \ldots, \rho_{n-2}, \delta ; k\right) \\
& \quad=\int_{0}^{\pi} \cos ^{2 \alpha-1}(\theta / 2) \sin ^{2 \beta-1}(\theta / 2) \prod_{j=1}^{(n-2)} \frac{\left[1-\rho_{j} \sin ^{2}(\theta / 2)\right]^{-\sigma_{j}}\left[1+\delta \cos ^{2}(\theta / 2)\right]^{-\gamma}}{\left(1-k^{2} \cos \theta\right)^{\mu+\frac{1}{2}}} d \theta,
\end{aligned}
$$

where $0 \leq k<1, \operatorname{Re}(\alpha)>O, \operatorname{Re}(\beta)>O ; \sigma_{j}(j=1, \ldots, n-2), \gamma, \mu \in C$;

$$
\max \left\{\left|\rho_{j}\right|,\left|\frac{\delta}{1+\delta}\right|,\left|\frac{2 k^{2}}{k^{2}-1}\right|\right\}<1 .
$$


In a recent paper, Saxena and Pathan [28] investigated an extension of equation (8) in the form:

$$
\begin{aligned}
& \Omega_{\left(\sigma_{1}, \ldots, \sigma_{\mu} ; \gamma ; L_{1}, \ldots, L_{n}\right)}^{(\alpha, \beta)}\left(\rho_{1}, \ldots, \rho_{\mu}, \delta ; \lambda_{1}, \ldots, \lambda_{n}\right) \\
& =\int_{0}^{\pi} \cos ^{2 \alpha-1}(\theta / 2) \sin ^{2 \beta-1}(\theta / 2) \prod_{i=1}^{m}\left[1-\rho_{i} \sin ^{2}(\theta / 2)\right]^{-\sigma_{i}} \\
& \quad \cdot\left[1+\delta \cos ^{2}(\theta / 2)\right]^{-\gamma} \prod_{j=1}^{n}\left(1-\lambda_{j}^{2} \cos \theta\right)^{-L_{j}} d \theta
\end{aligned}
$$

where $\min \{\operatorname{Re}(\alpha), \operatorname{Re}(\beta)\}>0 ;\left|\lambda_{j}\right|<1 ; \sigma_{i}, \gamma, L_{j} \in C$;

$$
\max \left\{\left|\rho_{i}\right|,\left|\frac{2 \lambda_{j}^{2}}{\lambda_{j}^{2}-1}\right|,\left|\frac{\delta}{1+\delta}\right|\right\}<1(i=1, \ldots, m ; j=1, \ldots, n) .
$$

In a recent paper [4], Chaurasia and Pandey investigated a new family of unified and generalized elliptic-type integrals:

$$
\begin{aligned}
& \bar{\Omega}_{\left(\lambda_{i}, L_{i}\right)}^{(\alpha, \beta)}\left(\left(\rho_{i}\right),\left(\delta_{i}\right) ; k_{j}\right)=\bar{\Omega}_{\left(\lambda_{1}, \ldots, \lambda_{n} ; L_{1}, \ldots, L_{M}\right)}^{(\alpha, \beta)}\left(\rho_{1}, \ldots, \rho_{N}, \delta_{1}, \ldots, \delta_{N} ; k_{1}, \ldots, k_{M}\right) \\
& =\int_{0}^{\pi} \cos ^{2 \alpha-1}(\theta / 2) \sin ^{2 \beta-1}(\theta / 2) \prod_{i=1}^{m}\left[1+\rho_{i} \sin ^{2}(\theta / 2)+\delta_{i} \cos ^{2}(\theta / 2)\right]^{-\lambda_{i}} \prod_{j=1}^{n}\left(1-k_{j}^{2} \cos \theta\right)^{-L_{j}} d \theta,
\end{aligned}
$$

where $\min \{\operatorname{Re}(\alpha), \operatorname{Re}(\beta)\}>0 ;\left|k_{j}\right|<1 ; \lambda_{i}, L_{i} \in C$;

$$
\max \left\{\left|\rho_{i}\right|,\left|\delta_{i}\right|,\left|\frac{2 k_{j}^{2}}{k_{j}^{2}-1}\right|,\left|\frac{\delta_{i}-\rho_{i}}{1+\delta_{i}}\right|\right\}<1(i=1, \ldots, N ; j=1, \ldots, M)
$$

which includes most of the known generalized and unified families of elliptic-type integrals (including those discussed in (1) through (9)). For more details also see [10], [25] and [29].

Upon a closer examination of the above equation (10), it can be seen that the family of elliptic-type integral $\bar{\Omega}_{\left(\lambda_{i}, L_{j}\right)}^{(\alpha, \beta)}\left(\left(\rho_{i}\right),\left(\delta_{i}\right) ; k_{j}\right)$ can be put in to the following form involving Eulertype integral:

$$
\begin{aligned}
& \bar{\Omega}_{\left(\lambda_{1}, \ldots, \lambda_{N} ; L_{1}, \ldots, L_{M}\right)}^{(\alpha, \beta)}\left(\rho_{1}, \ldots, \rho_{N}, \delta_{1}, \ldots, \delta_{N} ; k_{1}, \ldots, k_{M}\right) \\
& =\prod_{j=1}^{M}\left(1-k_{j}^{2}\right)^{-L_{j}} \prod_{i=1}^{N}\left(1+\delta_{i}\right)^{-\lambda_{i}} \int_{0}^{1} \omega^{\beta-1}(1-\omega)^{\alpha-1} \prod_{j=1}^{M}\left[1-\frac{2 \omega k_{j}^{2}}{k_{j}^{2}-1}\right]^{-L_{j}} \prod_{i=1}^{N}\left[1-\frac{\left(\delta_{i}-\rho_{i}\right) \omega}{\left(1+\delta_{i}\right)}\right]^{-\lambda_{i}} d \omega .
\end{aligned}
$$

A two-variable generating function $F(x, t)$ possess a formal (not necessarily convergent for $t \neq 0$ ) power series representation in $t$, can be written in the following form

$$
F(x, t)=\sum_{n=0}^{\infty} C_{n} f_{n}(x) t^{n}
$$


where each member of the generalized set $\left\{f_{n}(x)\right\}_{n=0}^{\infty}$ is independent of $x$ and $t$. The well known $\mathrm{H}$-function [32] is defined in the form

$$
H_{P_{\ell}, Q_{\ell}}^{M_{\ell}, N_{\ell}}\left[z \mid \begin{array}{l}
\left(a_{j}, A_{j}\right)_{1, P_{\ell}} \\
\left(b_{j}, B_{j}\right)_{1, Q_{\ell}}
\end{array}\right]=\frac{1}{2 \pi i} \int_{E} \theta(s) z^{s} d s,
$$

where

$$
\theta(s)=\frac{\prod_{j=1}^{M_{\ell}} \Gamma\left(b_{j}-B_{j} s\right) \prod_{j=1}^{N_{\ell}} \Gamma\left(1-a_{j}+A_{j} s\right)}{\prod_{j=M_{\ell}+1}^{Q_{\ell}} \Gamma\left(1-b_{j}+B_{j} s\right) \prod_{j=N_{\ell}+1}^{P_{\ell}} \Gamma\left(a_{j}-A_{j} s\right)} .
$$

$\ell=1, \ldots, r, i=\sqrt{-1}, z$ is not equal to zero but may be real or complex number and an empty product is interpreted as unity. $M_{\ell}, N_{\ell}, P_{\ell}, Q_{\ell}$ are non-negative integers satisfying $0 \leq N_{\ell} \leq P_{\ell}, O \leq M_{\ell} \leq Q_{\ell}$. The parameters $A_{1}, \ldots, A_{P_{\ell}} ; B_{1}, \ldots, B_{Q_{\ell}}$, are real positive numbers, $a_{1}, \ldots, a_{P_{\ell}} ; b_{1}, \ldots, b_{Q_{\ell}}$ are complex numbers such that the poles of the Gamma functions in the integrand in (13) do not coincide. $E$ is a suitable contour in the $s$-plane separating the poles of Gamma products with $+s$ and $-s$ in the numerator.

The H-function of several complex variables introduced by H. M. Srivastava and Rekha Panda [33, p.265] is represented and defined in the following manner:

$$
\begin{array}{r}
H_{0, \lambda:\left(u^{\prime}, v^{\prime}\right) ; \ldots ;\left(u^{r}, v^{r}\right)}^{A, C:\left[B^{\prime}\right]}\left[\begin{array}{l}
{\left[(a): \theta^{\prime}, \ldots, \theta^{r}\right]:\left[b^{\prime} ; \phi^{\prime}\right] ; \ldots ;\left[b^{r} ; \phi^{r}\right] ;} \\
{\left[(c): \psi^{\prime}, \ldots, \psi^{r}\right]:\left[d^{\prime} ; \delta^{\prime}\right] ; \ldots ;\left[d^{r}, \delta^{r}\right] ;}
\end{array} Z_{1}, \ldots, Z_{r}\right] \\
\quad=\frac{1}{(2 \pi i)^{r}} \int_{E_{1}} \cdots \int_{E_{r}} R_{1}\left(s_{1}\right) \cdots R_{r} s_{r} T\left(s_{1}, \ldots, s_{r}\right) z_{1}^{s_{1}} \cdots z_{r}^{s_{r}} d s_{1} \cdots d s_{r},
\end{array}
$$

where

$$
\begin{aligned}
R_{i}\left(s_{i}\right)= & \frac{\prod_{j=1}^{u^{(i)}} \Gamma\left(d_{j}^{i}-\delta_{j}^{i} s_{i}\right) \prod_{j=1}^{v^{i}} \Gamma\left(1-b_{j}^{i}+\phi_{j}^{i} s_{i}\right)}{\prod_{j=u^{i}+1}^{D^{i}} \Gamma\left(1-d_{j}^{i}+\delta_{j}^{i} s_{i}\right) \prod_{j=\nu^{i}+1}^{B^{i}} \Gamma\left(b_{j}^{i}-\phi_{j}^{i} s_{i}\right)}, \quad \forall i=1, \ldots, r \\
T\left(s_{1}, \ldots, s_{r}\right)= & \frac{\prod_{j=1}^{\lambda} \Gamma\left(1-a_{j}+\sum_{i=1}^{r} \theta_{j}^{i} s_{i}\right)}{\prod_{j=\lambda+1}^{A} \Gamma\left(a_{j}-\sum_{i=1}^{r} \theta_{j}^{i} s_{i}\right) \prod_{j=1}^{C} \Gamma\left(1-c_{j}+\sum_{i=1}^{r} \psi_{j}^{i} s_{i}\right)}
\end{aligned}
$$

$i=\sqrt{-1}$, an empty product is interpreted as unity, the coefficients $\theta_{j}^{i}, j=1, \ldots, A ; \phi_{j}^{i}, j=$ $1, \ldots, B^{i} ; \psi_{j}^{i}, j=1, \ldots, C ; \delta_{j}^{i}, j=1, \ldots, D^{i} \forall i=1, \ldots, r$ are positive numbers and $\lambda, u^{i}, v^{i}, A, B^{i}$, $C, D^{i}$ are integers such that $0 \leq \lambda \leq A, 1 \leq u^{i} \leq D^{i}, C \geq 0$ and $0 \leq V^{i} \leq B^{i} \forall i=1, \ldots, r$. The 
contour $E_{i}$ in the complex $s_{i}$-plane is of the Mellin-Barnes type which runs from $-i \infty$ to $+i \infty$ with indentations, if necessary, in such a manner that all poles of $\Gamma\left(d_{j}^{i}-\delta_{j}^{i} s_{i}\right), j=1, \ldots, u^{i}$, are to the right and those of $\Gamma\left(1-b_{j}^{i}+\phi_{j}^{i} s_{i}\right), j=1, \ldots, v^{i}$ and $\Gamma\left(1-a_{j}+\sum_{i=1}^{r} \theta_{j}^{i} s_{i}\right), j=1, \ldots, \lambda$, to the left of $s_{i}$, the various parameters being so restricted that these poles are all simple and none of them coincide and with the points $z_{i} ; i=1, \ldots, r$ being tacitly excluded. When $A_{j}$ $\left(j=1, \ldots, P_{\ell}\right)=B_{J}\left(j=1, \ldots, Q_{\ell}\right)=1$ in (13), it reduces to a Meijer's G-function [32].

If we take $\theta^{\prime}, \ldots, \theta^{r}=\phi^{\prime}, \ldots, \phi^{r}=\psi^{\prime}, \ldots, \psi^{r}=\delta^{\prime}, \ldots, \delta^{r}=1$ in (14), we have the following interesting transformation:

$$
\begin{aligned}
& H_{A, C:\left[B^{\prime}, D^{\prime}\right] ; \ldots ;\left[B^{r}, D^{r}\right]}^{0, \lambda:\left(\left(u^{\prime}, v^{\prime}\right) \ldots\left(u^{r}, v^{r}\right)\right.}\left[\begin{array}{l}
{[(a): 1, \ldots, 1]:\left[b^{\prime} ; 1\right] ; \ldots ;\left[b^{r} ; 1\right] ;} \\
{[(c): 1, \ldots, 1]:\left[d^{\prime} ; 1\right] ; \ldots ;\left[d^{r}, 1\right] ;}
\end{array} Z_{1}, \ldots, Z_{r}\right] \\
& \quad=G_{A, C:\left[B^{\prime}, D^{\prime}\right] ; \ldots ;\left[B^{r}, D^{r}\right]}^{0, \lambda:\left(u^{\prime}, v^{\prime}\right) \ldots ;\left(u^{r}, v^{r}\right)}\left[\begin{array}{c}
{\left[(a): b, \ldots, b^{r}\right] ;} \\
{\left[(c): d^{\prime}, \ldots, d^{r}\right] ;}
\end{array} Z_{1}, \ldots, Z_{r}\right] .
\end{aligned}
$$

In this article we have studied two new theorems associated with two variables generating functions. Beside generalizing most of the known elliptic-type integrals some new families of elliptio-type integrals can also be deduced with the help of the results obtained in this article. Such generalized and new families of elliptictype integrals play an important role in evaluation of a number of Euler-type integrals involving various generating functions. The basic idea of evolving the theorems discussed in this article is inspired by the research work of Mohammed [22], Saran [24] and Srivastava and Yeh [36].

\section{Theorems}

In this section we derive two new theorems and their corollaries on generating functions associated with the families of elliptic-type integrals. These theorems and corollaries can be used to establish various known and new elliptic type integrals. Some of the significant applications of the results derived in this section are discussed in the Section 3.

Theorem 1. Let the generating function $F(x, t)$ and $H_{P_{\ell}, Q_{\ell}}^{M_{\ell}, N_{\ell}}(z)$ be the H-function of one variable defined in (12) and (13) respectively. Then

$$
\begin{aligned}
& \int_{0}^{1} \omega^{\alpha-1}(1-\omega)^{\gamma-\alpha-1} \prod_{\ell=1}^{r} H_{P_{\ell}, Q_{\ell}}^{M_{\ell}, N_{\ell}}\left[\left(\omega z_{\ell}\right)^{\xi_{\ell}} \mid \begin{array}{c}
\left(a_{j}^{\ell}, A_{j}^{\ell}\right)_{1, P_{\ell}} \\
\left(b_{j}^{\ell}, B_{j}^{\ell}\right)_{1, Q_{\ell}}
\end{array}\right] F\left[x, t \omega^{\eta}(1-\omega)^{\mu}\right] d \omega \\
& =\Gamma(\gamma-\alpha) \sum_{n=0}^{\infty}(\gamma-\alpha)_{\mu n} C_{n} f_{n}(x) t^{n} \\
& \cdot H_{A+1, C+1:\left[P_{1}, Q_{1}\right] ; \ldots ;\left[P_{r}, Q_{r}\right]}^{0, \lambda+1:\left(M_{1}, N_{1}\right) ; ;\left(M_{r}, N_{r}\right)}\left[\begin{array}{l}
{\left[(1-\alpha-\eta n): \xi_{1}, \ldots, \xi_{r}\right]:\left[a_{j}^{\prime} ; A_{j}^{\prime}\right] ; \ldots ;\left[a_{j}^{r} ; A_{j}^{r}\right] ;} \\
\left.\left[(1-\gamma-\eta n-\mu n): \xi_{1}, \ldots, \xi_{r}\right]:\left[b_{j}^{\prime} ; B_{j}^{\prime}\right] ; \ldots ;\left[b_{j}^{r}, B_{j}^{r}\right] ; Z_{1}^{\xi_{1}}, \ldots, Z_{r}^{\xi_{r}}\right],(18
\end{array}\right]
\end{aligned}
$$

provided that $\min \{\operatorname{Re}(\alpha), \operatorname{Re}(\gamma-\alpha)\}>O, \operatorname{Re}(\eta)>O, \operatorname{Re}(\mu)>0$ and $\max \left|z_{\ell}\right| \rightarrow 0(\ell=1, \ldots, r)$ and $\min \left|z_{\ell}\right| \rightarrow \infty(\ell=1 \ldots, r)$. 
On taking $\xi_{\ell}=1$ (for $\ell=1, \ldots, r$ ), Theorem 1 gives the following corollary:

Corollary 1. Let the generating function $F(x, t)$ and $H_{P_{\ell}, Q_{\ell}}^{M_{\ell}, N_{\ell}}(z)$ be the H-function of one variable defined in (12) and (13) respectively. Then

$$
\begin{aligned}
& \int_{0}^{1} \omega^{\alpha-1}(1-\omega)^{\gamma-\alpha-1} \prod_{\ell=1}^{r} H_{P_{\ell}, Q_{\ell}}^{M_{\ell}, N_{\ell}}\left[\left(\omega z_{\ell}\right) \mid \begin{array}{c}
\left(a_{j}^{\ell}, A_{j}^{\ell}\right)_{1, P_{\ell}} \\
\left(b_{j}^{\ell}, B_{j}^{\ell}\right)_{1, Q_{\ell}}
\end{array}\right] F\left[x, t \omega^{\eta}(1-\omega)^{\mu}\right] d \omega \\
& =\Gamma(\gamma-\alpha) \sum_{n=0}^{\infty}(\gamma-\alpha)_{\mu n} C_{n} f_{n}(x) t^{n} \\
& \cdot H_{A+1, C+1:\left[P_{1}, Q_{1}\right] ; \ldots ;\left[P_{r}, Q_{r}\right]}^{0, \lambda+1:\left(M_{1}, N_{1}\right) ; \ldots ;\left(M_{r}, N_{r}\right)}\left[\begin{array}{l}
(1-\alpha-\eta n):\left[a_{j}^{\prime} ; A_{j}^{\prime}\right] ; \ldots ;\left[a_{j}^{r} ; A_{j}^{r}\right] ; \\
(1-\gamma-\eta n-\mu n):\left[b_{j}^{\prime} ; B_{j}^{\prime}\right] ; \ldots ;\left[b_{j}^{r}, B_{j}^{r}\right] ; Z_{1}, \ldots, Z_{r}
\end{array}\right],
\end{aligned}
$$

valid under the conditions as given in (18).

On taking $\xi_{1}=\cdots=\xi_{r}=A_{j}^{\prime}=\cdots=A_{j}^{r}=B_{j}^{\prime}=\cdots=B_{j}^{r}=\eta=\mu=1$, Theorem 1 reduces in the following corollary:

Corollary 2. Let the generating function $F(x, t)$ and $H_{P_{\ell}, Q_{\ell}}^{M_{\ell}, N_{\ell}}(z)$ be the H-function of one variable defined in (12) and (13) respectively. Then

$$
\begin{aligned}
& \int_{0}^{1} \omega^{\alpha-1}(1-\omega)^{\gamma-\alpha-1} \prod_{\ell=1}^{r} H_{P_{\ell}, Q_{\ell}}^{M_{\ell}, N_{\ell}}\left[\left(\omega z_{\ell}\right) \mid \begin{array}{c}
\left(a_{j}^{\ell}, A_{j}^{\ell}\right)_{1, P_{\ell}} \\
\left(b_{j}^{\ell}, B_{j}^{\ell}\right)_{1, Q_{\ell}}
\end{array}\right] F[x, t \omega(1-\omega)] d \omega \\
& =\Gamma(\gamma-\alpha) \sum_{n=0}^{\infty}(\gamma-\alpha)_{n} C_{n} f_{n}(x) t^{n} \\
& \cdot G_{A+1, C+1:\left[P_{1}, Q_{1}\right] ; \ldots ;\left[P_{r}, Q_{r}\right]}^{0, \lambda+1:\left(M_{1}, N_{1}\right) ; \ldots ;\left(M_{r}, N_{r}\right)}\left[\begin{array}{l}
{\left[(1-\alpha-n): a_{j}^{\prime} ; a_{j}^{\prime}\right]} \\
{\left[(1-\gamma-2 n): b_{j}^{\prime}, \ldots, \beta_{j}^{r}\right] ; Z_{1}, \ldots, Z_{r}}
\end{array}\right],
\end{aligned}
$$

where $G_{A+1, C+1}^{0, \lambda+1}\left[z_{1}, \ldots, z_{r}\right]$ involved in (20) is the Meijer's G-function of r-variables [16].

Now we state an another modification of the Theorem 1, which can be used to obtain various (new or known) interesting generalization of elliptic-type integrals.

Theorem 2. Let the generating function function $F(x, t)$ and $H_{P_{\ell}, Q_{\ell}}^{M_{\ell}, N_{\ell}}(z)$ be the H-function of one variable defined in (12) and (13) respectively. Then

$$
\begin{aligned}
\int_{0}^{1} \omega^{\beta-1}(1-\omega)^{\alpha-1} \prod_{\ell=1}^{r} H_{P_{\ell}, Q_{\ell}}^{M_{\ell}, N_{\ell}}\left[\left(\omega z_{\ell}\right)^{\xi_{\ell}} \mid \begin{array}{c}
\left(a_{j}^{\ell}, A_{j}^{\ell}\right)_{1, P_{\ell}} \\
\left(b_{j}^{\ell}, B_{j}^{\ell}\right)_{1, Q_{\ell}}
\end{array}\right] \\
\quad \cdot \prod_{j=1}^{M}\left[1-\frac{2 \omega k_{j}^{2}}{k_{j}^{2}-1}\right]^{-L_{j}} \prod_{i=1}^{N}\left[1-\frac{\left(\delta_{i}-\rho_{i}\right) \omega}{\left(1+\delta_{i}\right)}\right]^{-\lambda_{i}} F\left[x, t \omega^{\eta}(1-\omega)^{\mu}\right] d \omega \\
=T_{1} T_{2} \Gamma(\alpha) \sum_{n=0}^{\infty} C_{n} f_{n}(x) t^{n}(\alpha)_{\mu n} H_{A+1, C+1:\left[P_{1}, Q_{1}\right] ; \ldots ;\left[P_{r}, Q_{r}\right]}^{\left.0, \lambda+1:\left(M_{1}, N_{1}\right) ; \ldots ; M_{r}, N_{r}\right)}
\end{aligned}
$$




$$
\cdot\left[\begin{array}{lr}
{\left[(1-\beta-\eta n): \xi_{1}, \ldots, \xi_{r}, 1, \ldots, 1,1, \ldots, 1\right]} & :\left[a_{j}^{\prime} ; A_{j}^{\prime}\right] ; \ldots ;\left[a_{j}^{r} ; A_{j}^{r}\right] ; \\
{\left[(1-\beta-\alpha-\eta n-\mu n): \xi_{1}, \ldots, \xi_{r}, 1, \ldots, 1,1, \ldots, 1\right]:\left[b_{j}^{\prime} ; B_{j}^{\prime}\right] ; \ldots ;\left[b_{j}^{r} ; B_{j}^{r}\right] ;}
\end{array}\right.
$$

where

$$
\begin{aligned}
& T_{1}=\prod_{j=1}^{M}\left[1-\frac{2 k_{j}^{2}}{k_{j}^{2}-1}\right]^{-L_{j}} \\
& T_{2}=\prod_{i=1}^{N}\left[1-\frac{\left(\delta_{i}-\rho_{i}\right)}{\left(1+\delta_{i}\right)}\right]^{-\lambda_{j}}
\end{aligned}
$$

provided that

$\min \{\operatorname{Re}(\alpha), \operatorname{Re}(\beta)\}>0, \operatorname{Re}(\eta)>0, \operatorname{Re}(\mu)>0, \delta_{i}, \rho_{i}, \lambda_{i}, L_{j} \in C ;\left|k_{j}\right|<1,(i=1, \ldots, N, j=1, \ldots, M)$, $\max \left\{\left|\rho_{i}\right|,\left|\delta_{i}\right|,\left|\frac{2 k_{j}^{2}}{k_{j}^{2}-1}\right|,\left|\frac{\delta_{i}-\rho_{i}}{1+\delta_{i}}\right|\right\}<1, \max \left|z_{\ell}\right| \rightarrow 0,(\ell=1, \ldots, r)$ and $\min \left|z_{\ell}\right| \rightarrow \infty(\ell=1, \ldots, r)$.

On taking $\xi_{\ell}=1(\ell=1, \ldots, r)$, we obtain the following corollary:

Corollary 3. Let the generating function $F(x, t)$ and $H_{P_{\ell}, Q_{\ell}}^{M_{\ell}, N_{\ell}}(z)$ be the H-function of one variable defined in (12) and (13) respectively. Then

$$
\begin{aligned}
\int_{0}^{1} \omega^{\beta-1}(1-\omega)^{\alpha-1} \prod_{\ell=1}^{r} H_{P_{\ell}, Q_{\ell}}^{M_{\ell}, N_{\ell}}\left[\left(\omega z_{\ell}\right) \mid \begin{array}{c}
\left(a_{j}^{\ell}, A_{j}^{\ell}\right)_{1, P_{\ell}} \\
\left(b_{j}^{\ell}, B_{j}^{\ell}\right)_{1, Q_{\ell}}
\end{array}\right] \\
\cdot \prod_{j=1}^{M}\left[1-\frac{2 \omega k_{j}^{2}}{k_{j}^{2}-1}\right]^{-L_{j}} \prod_{i=1}^{N}\left[1-\frac{\left(\delta_{i}-\rho_{i}\right) \omega}{\left(1+\delta_{i}\right)}\right]^{-\lambda_{i}} F\left[x, t \omega^{\eta}(1-\omega)^{\mu}\right] d \omega \\
=T_{1} T_{2} \Gamma(\alpha) \sum_{n=0}^{\infty} C_{n} f_{n}(x) t^{n}(\alpha)_{\mu n} H_{A+1, C+1:\left[P_{1}, Q_{1}\right] ; \ldots ;\left[P_{r}, Q_{r}\right]}^{0, \lambda+1:\left(M_{1}, N_{1}\right) ; \ldots ;\left(M_{r}, N_{r}\right)} \\
\cdot\left[\begin{array}{l}
{\left[(1-\beta-\eta n):\left[a_{j}^{\prime} ; A_{j}^{\prime}\right] ; \ldots ;\left[a_{j}^{r} ; A_{j}^{r}\right] ;\right.} \\
{\left[(1-\beta-\alpha-\eta n-\mu n):\left[b_{j}^{\prime} ; B_{j}^{\prime}\right] ; \ldots ;\left[b_{j}^{r} ; B_{j}^{r}\right] ; Z_{1}, \ldots, Z_{r}\right],}
\end{array}\right.
\end{aligned}
$$

valid under the condition as needed for (21).

Particularly, on taking $\xi_{1}=\cdots=\xi_{r}=A_{j}^{\prime}=\cdots=A_{j}^{r}=B_{j}^{\prime}=\cdots=B_{j}^{r}=\eta=\mu=1 ; \delta_{i}=\rho_{i} ;$ $\lambda_{i}=0$ for $(1, \ldots, N-1), \rho_{N}=O ; \delta_{N}=\delta, \lambda_{n}=\gamma$, following corollary can be stated:

Corollary 4. Let the generating function $F(x, t)$ and $H_{P_{\ell}, Q_{\ell}}^{M_{\ell}, N_{\ell}}(z)$ be the H-function of one variable defined in (12) and (13) respectively. Then

$$
\int_{0}^{1} \omega^{\beta-1}(1-\omega)^{\alpha-1} \prod_{\ell=1}^{r} H_{P_{\ell}, Q_{\ell}}^{M_{\ell}, N_{\ell}}\left[\left(\omega z_{\ell}\right) \mid \begin{array}{l}
\left(a_{j}^{\ell}, A_{j}^{\ell}\right)_{1, P_{\ell}} \\
\left(b_{j}^{\ell}, B_{j}^{\ell}\right)_{1, Q_{\ell}}
\end{array}\right]
$$




$$
\begin{aligned}
& \cdot \prod_{j=1}^{M}\left[1-\frac{2 \omega k_{j}^{2}}{k_{j}^{2}-1}\right]^{-L_{j}}\left[1-\frac{\delta \omega}{1+\delta}\right]^{-\gamma} F[x, t \omega(1-\omega)] d \omega \\
= & W_{1} W_{2} \Gamma(\alpha) \sum_{n=0}^{\infty} C_{n} f_{n}(x) t^{n}(\alpha)_{n} G_{A+1, C+1:\left[P_{1}, Q_{1}\right] ; \ldots ;\left[P_{r}, Q_{r}\right]}^{0, \lambda+1:\left(M_{1}, N_{1}\right) ;\left(M_{r}, N_{r}\right)} \\
& \cdot\left[\begin{array}{l}
{\left[(1-\beta-n): a_{j}^{\prime} ; a_{j}^{r} ;\right.} \\
{\left[(1-\beta-\alpha-2 n): b_{j}^{\prime} ; b_{j}^{r} ;\right.}
\end{array} Z_{1}, \ldots, Z_{r}\right],
\end{aligned}
$$

where

$$
\begin{aligned}
& W_{1}=\prod_{j=1}^{M}\left[1-\frac{2 k_{j}^{2}}{k_{j}^{2}-1}\right]^{-L_{j}} \\
& W_{2}=\left[1-\frac{\delta}{1+\delta}\right]^{-\gamma}
\end{aligned}
$$

provided that

$$
\operatorname{Re}(\alpha), \operatorname{Re}(\beta)>O, L_{j}, \gamma \in C ;\left|k_{j}\right|<1, \max \left\{\left|\frac{2 k_{j}^{2}}{k_{j}^{2}-1}\right|,\left|\frac{\delta}{1+\delta}\right|\right\}<1,(j=1, \ldots, M i=1, \ldots, N) .
$$

Proofs. Expressing $F(x, t)$ by its power series from (12) in the integral (18), changing the order of integration and summation, which is permissible, due to uniform convergence of the series involved. Using the definition (14) of the $\mathrm{H}$-function of several variables in the evaluation of the resulting integral, we get the result (18), which proves Theorem 1.

The proofs of Theorem 2 and Corollaries 1, 2, 3 and 4 are similar to that of the Theorem 1 .

\section{Applications}

In view of the importance and usefulness of the theorems and corollaries discussed in the last section, we mention some interesting applications, which indicates manifold generality of the results obtained in this article.

(I) Consider the generating function [34]

$$
F(x, t)=(1-x)^{-\sigma} \sum_{n=0}^{\infty}(\sigma)_{n} \frac{x^{n} t^{n}}{n !}
$$

and by the use of the Theorem 1, under the conditions stated in (18), we get the following interesting results:

$$
\int_{0}^{1} \omega^{\alpha-1}(1-\omega)^{\gamma-\alpha-1} \prod_{\ell=1}^{r} H_{P_{\ell}, Q_{\ell}}^{M_{\ell}, N_{\ell}}\left[\left(\omega z_{\ell}\right)^{\xi_{\ell}} \mid \begin{array}{c}
\left(a_{j}^{\ell}, A_{j}^{\ell}\right)_{1, P_{\ell}} \\
\left(b_{j}^{\ell}, B_{j}^{\ell}\right)_{1, Q_{\ell}}
\end{array}\right]\left[1-x t \omega^{\eta}(1-\omega)^{\mu}\right]^{-\sigma} d \omega
$$




$$
\begin{gathered}
=\Gamma(\gamma-\alpha) \sum_{n=0}^{\infty}(\gamma-\alpha)_{\mu n}(\sigma)_{n} \frac{x^{n} t^{n}}{n !} \\
\cdot H_{A+1, C+1:\left[P_{1}, Q_{1}\right] ; \ldots ;\left[P_{r}, Q_{r}\right]}^{0, \lambda+1:\left(M_{1}, N_{1}\right) ; ;\left(M_{r}, N_{r}\right)}\left[\begin{array}{l}
{\left[(1-\alpha-\eta n): \xi_{1}, \ldots, \xi_{r}\right]:\left[a_{j}^{\prime} ; A_{j}^{\prime}\right] ; \ldots ;\left[a_{j}^{r} ; A_{j}^{r}\right] ;} \\
{\left[(1-\gamma-\eta n-\mu n): \xi_{1}, \ldots, \xi_{r}\right]:\left[b_{j}^{\prime} ; B_{j}^{\prime}\right] ; \ldots ;\left[b_{j}^{r}, B_{j}^{r}\right] ; Z_{1}^{\xi_{1}}, \ldots, Z_{r}^{\xi_{r}}}
\end{array}\right],
\end{gathered}
$$

which on setting $\omega=\cos ^{2}(\theta / 2)$ and $\cos \theta=2 \cos ^{2}(\theta / 2)-1$, the above equation (26) gives the following generalization of the elliptic-type integral

$$
\begin{aligned}
& \int_{0}^{1} \cos ^{2 \alpha-1}\left(\frac{\theta}{2}\right) \sin ^{2 \gamma-2 \alpha-1}\left(\frac{\theta}{2}\right) \prod_{\ell=1}^{r} H_{P_{\ell}, Q_{\ell}}^{M_{\ell}, N_{\ell}}\left[\left(\cos ^{2}\left(\frac{\theta}{2}\right) z_{\ell}\right)^{\xi_{\ell}} \mid \begin{array}{c}
\left(a_{j}^{\ell}, A_{j}^{\ell}\right)_{1, P_{\ell}} \\
\left(b_{j}^{\ell}, B_{j}^{\ell}\right)_{1, Q_{\ell}}
\end{array}\right] \\
& \cdot\left[1-x t \cos ^{2 \eta}\left(\frac{\theta}{2}\right) \sin ^{2 \mu}\left(\frac{\theta}{2}\right)\right]^{-\sigma} d \theta \\
& =\Gamma(\gamma-\alpha) \sum_{n=0}^{\infty}(\gamma-\alpha)_{\mu n}(\sigma)_{n} \frac{x^{n} t^{n}}{n !} \\
& \cdot H_{A+1, C+1:\left[P_{1}, Q_{1}\right] ; \ldots ;\left[P_{r}, Q_{r}\right]}^{\left.0, \lambda+1:\left(M_{1}, N_{1}\right) ; \ldots ; M_{r}, N_{r}\right)}\left[\begin{array}{l}
{\left[(1-\alpha-\eta n): \xi_{1}, \ldots, \xi_{r}\right]:\left[a_{j}^{\prime} ; A_{j}^{\prime}\right] ; \ldots ;\left[a_{j}^{r} ; A_{j}^{r}\right] ;} \\
\left.\left[(1-\gamma-\eta n-\mu n): \xi_{1}, \ldots, \xi_{r}\right]:\left[b_{j}^{\prime} ; B_{j}^{\prime}\right] ; \ldots ;\left[b_{j}^{r}, B_{j}^{r}\right] ; Z_{1}^{\xi_{1}}, \ldots, Z_{r}^{\xi_{r}}\right] .
\end{array}\right.
\end{aligned}
$$

On setting $\omega=\sin ^{2}(\theta / 2)$ and using $\cos \theta=1-2 \sin ^{2}(\theta / 2), \sigma \rightarrow 0$ in (26), we get the following generalized family of elliptic-type integrals:

$$
\begin{aligned}
& \int_{0}^{1} \sin ^{2 \alpha-1}\left(\frac{\theta}{2}\right) \cos ^{2 \gamma-2 \alpha-1}\left(\frac{\theta}{2}\right) \prod_{\ell=1}^{r} H_{P_{\ell}, Q_{\ell}}^{M_{\ell}, N_{\ell}}\left[\left(\sin ^{2}\left(\frac{\theta}{2}\right) z_{\ell}\right)^{\xi_{\ell}} \mid \begin{array}{l}
\left(a_{j}^{\ell}, A_{j}^{\ell}\right)_{1, P_{\ell}} \\
\left(b_{j}^{\ell}, B_{j}^{\ell}\right)_{1, Q_{\ell}}
\end{array}\right] d \theta \\
& =\Gamma(\gamma-\alpha) \sum_{n=0}^{\infty}(\gamma-\alpha)_{\mu n}(\sigma)_{n} \frac{x^{n} t^{n}}{n !} \\
& \cdot H_{A+1, C+1:\left[P_{1}, Q_{1}\right] ; \ldots ;\left[P_{r}, Q_{r}\right]}^{\left.0, \lambda+\left(M_{1}, N_{1}\right) ; \ldots ; M_{r}, N_{r}\right)}\left[\begin{array}{l}
{\left[(1-\alpha): \xi_{1}, \ldots, \xi_{r}\right]:\left[a_{j}^{\prime} ; A_{j}^{\prime}\right] ; \ldots ;\left[a_{j}^{r} ; A_{j}^{r}\right] ; Z_{1}^{\xi_{1}}, \ldots, Z_{r}^{\xi_{r}}} \\
{\left[(1-\gamma): \xi_{1}, \ldots, \xi_{r}\right]:\left[b_{j}^{\prime} ; B_{j}^{\prime}\right] ; \ldots ;\left[b_{j}^{r}, B_{j}^{r}\right] ;}
\end{array}\right] .
\end{aligned}
$$

It can be seen that the above elliptic-type integral (26) also provides generalization to a number of known elliptic-type integrals and the well known complete elliptic-type integrals [3] of the first kind.

Also, by the application of the Theorem 2, under the conditions stated for (21) and generating function (25), we have obtained the following new family of elliptio-type integrals, which also generalizes a number of known families of elliptic - type integrals

$$
\begin{aligned}
& \int_{0}^{1} \omega^{\beta-1}(1-\omega)^{\alpha-1} \prod_{\ell=1}^{r} H_{P_{\ell}, Q_{\ell}}^{M_{\ell}, N_{\ell}}\left[\left(\omega z_{\ell}\right)^{z_{\ell}} \mid \begin{array}{c}
\left(a_{j}^{\ell}, A_{j}^{\ell}\right)_{1, P_{\ell}} \\
\left(b_{j}^{\ell}, B_{j}^{\ell}\right)_{1, Q_{\ell}}
\end{array}\right] \\
& \quad \cdot \prod_{j=1}^{M}\left[1-\frac{2 \omega k_{j}^{2}}{k_{j}^{2}-1}\right]^{-L_{j}} \prod_{i=1}^{N}\left[1-\frac{\left(\delta_{i}-\rho_{i}\right) \omega}{\left(1+\delta_{i}\right)}\right]^{-\lambda_{i}}\left[1-x t \omega^{\eta}(1-\omega)^{\mu}\right]^{-\sigma} d \omega
\end{aligned}
$$




$$
\begin{aligned}
& =T_{1} T_{2} \Gamma(\alpha) \sum_{n=0}^{\infty}(\alpha)_{\mu n}(\sigma)_{n} \frac{x^{n} t^{n}}{n !} H_{A+1, C+1:\left[P_{1}, Q_{1}\right] ; \ldots ;\left[P_{r}, Q_{r}\right]}^{0, \lambda+1:\left(M_{1}, N_{1} ; \ldots ;\left(M_{r}, N_{r}\right)\right.} \\
& \cdot\left[\begin{array}{l}
{\left[(1-\beta-\eta n): \xi_{1}, \ldots, \xi_{r}, 1, \ldots, 1,1, \ldots, 1\right]:\left[a_{j}^{\prime} ; A_{j}^{\prime}\right] ; \ldots ;\left[a_{j}^{r} ; A_{j}^{r}\right] ;} \\
{\left[(1-\beta-\alpha-\eta n-\mu n): \xi_{1}, \ldots, \xi_{r}, 1, \ldots, 1,1, \ldots, 1\right]:\left[b_{j}^{\prime} ; B_{j}^{\prime}\right] ; \ldots ;\left[b_{j}^{r}, B_{j}^{r}\right] ;}
\end{array}{\left(Z,{ }^{2}\right)^{\xi_{1}}, \ldots,\left(Z_{r}\right)^{\xi_{r}}}^{\xi_{r}} .\right.
\end{aligned}
$$

For $\sigma \rightarrow 0$ and $\omega=\sin ^{2}\left(\frac{\theta}{2}\right)$ with $\xi_{1}=\cdots=\xi_{r}=A_{j}^{\prime}=\cdots=A_{j}^{r}=B_{j}^{\prime}=\cdots=B_{j}^{r}=1$, above (29) yields the following explicit representation of generalized family of elliptic-type integral

$$
\begin{gathered}
\int_{0}^{1} \cos ^{2 \alpha-1}\left(\frac{\theta}{2}\right) \sin ^{2 \beta-1}\left(\frac{\theta}{2}\right) \prod_{\ell=1}^{r} H_{P_{\ell}, Q_{\ell}}^{M_{\ell}, N_{\ell}}\left[\left(\sin ^{2}\left(\frac{\theta}{2}\right) z_{\ell}\right) \mid \begin{array}{l}
\left(a_{j}^{\ell} ; 1\right)_{1, P_{\ell}} \\
\left(b_{j}^{\ell} ; 1\right)_{1, Q_{\ell}}
\end{array}\right] \\
\cdot \prod_{j=1}^{M}\left[1-\frac{2 \sin ^{2}\left(\frac{\theta}{2}\right) k_{j}^{2}}{k_{j}^{2}-1}\right]^{-L_{j}} \prod_{i=1}^{N}\left[1-\frac{\left(\delta_{i}-\rho_{i}\right) \sin ^{2}\left(\frac{\theta}{2}\right)}{\left(1+\delta_{i}\right)}\right]^{-\lambda_{i}} \\
=T_{1} T_{2} G_{\left.A+1, C+1:\left[P_{1}, Q_{1}\right] ; \ldots ; P_{r}, Q_{r}\right]}^{0, \lambda+1:\left(M_{1}, N_{1}\right) ; \ldots ;\left(M_{r}, N_{r}\right)}\left[\begin{array}{l}
{\left[(1-\beta): a_{j}^{\prime} ; \ldots ; a_{j}^{r} ;\right.} \\
{\left[(1-\alpha-\beta): b_{j}^{\prime} ; \ldots ; b_{j}^{r} ; Z_{1}, \ldots, Z_{r}\right] .}
\end{array}\right.
\end{gathered}
$$

also for $\sigma \rightarrow 0$ and $\omega=\sin ^{2}\left(\frac{\theta}{2}\right)$ with $\xi_{1}=\cdots=\xi_{r}=A_{j}^{\prime}=\cdots=A_{j}^{r}=B_{j}^{\prime}=\cdots=B_{j}^{r}=1 ;\left(z_{\ell}\right)^{\xi_{\ell}}=y_{\ell}$; for $\ell=1,2, \ldots, r ; \delta_{i}=\rho_{i}=0$ or $i=l, 2, \ldots,(N-1)$ and $\rho_{N}=0 ; \delta_{N}=\delta ; \lambda_{i}=\gamma$, above (29) yields the following explicit representation of generalized family of elliptic-type integral

$$
\begin{gathered}
\int_{0}^{\pi} \cos ^{2 \alpha-1}\left(\frac{\theta}{2}\right) \sin ^{2 \beta-1}\left(\frac{\theta}{2}\right) \prod_{\ell=1}^{r} H_{P_{\ell}, Q_{\ell}}^{M_{\ell}, N_{\ell}}\left[\left(\sin ^{2}\left(\frac{\theta}{2}\right) y_{\ell}\right) \mid \begin{array}{l}
\left(a_{j}^{\ell} ; 1\right)_{1, P_{\ell}} \\
\left(b_{j}^{\ell} ; 1\right)_{1, Q_{\ell}}
\end{array}\right] \\
\cdot \prod_{j=1}^{M}\left[1-\frac{2 k_{j}^{2} \sin ^{2}\left(\frac{\theta}{2}\right)}{k_{j}^{2}-1}\right]^{-L_{j}} \prod_{i=1}^{N}\left[1-\frac{\delta\left(\sin ^{2} \frac{\theta}{2}\right)}{1+\delta}\right]^{-\gamma} d \theta \\
=\Gamma(\alpha) W_{1} W_{2} G_{A+1, C+1}^{0, \lambda+1}\left[\begin{array}{l}
{\left[(1-\beta-n): a_{j}^{\prime} ; \ldots ; a_{j}^{r} ;\right.} \\
{\left[(1-\alpha-\beta-n): b_{j}^{\prime} ; \ldots ; b_{j}^{r} ; y_{1}, \ldots, y_{r}\right] .}
\end{array}\right.
\end{gathered}
$$

(II) Consider the generating relation [34]

$$
F(x, t)=\left(1-X_{1} t\right)^{-\alpha_{1}}\left(1-X_{2} t\right)^{-\alpha_{2}}=\sum_{n=0}^{\infty} g_{n}^{\alpha_{1}, \alpha_{2}}\left(X_{1}, X_{2}\right) t^{n}
$$

where $g_{n}^{\alpha_{1}, \alpha_{2}}$ the Lagrange polynomial defined by

$$
g_{n}^{\alpha_{1}, \alpha_{2}}(x, y)=\sum_{n=0}^{\infty} \frac{\left(\alpha_{1}\right)_{r}\left(\alpha_{2}\right)_{n-r}}{r !(n-r) !} x^{r} y^{n-r},
$$

and by the application of Theorem 1, under the conditions stated in (18), we get

$$
\int_{0}^{1} \omega^{\beta-1}(1-\omega)^{\gamma-\alpha-1} \prod_{\ell=1}^{r} H_{P_{\ell}, Q_{\ell}}^{M_{\ell}, N_{\ell}}\left[\left(\omega z_{\ell}\right)^{z_{\ell}} \mid \begin{array}{l}
\left(a_{j}^{\ell}, A_{j}^{\ell}\right)_{1, P_{\ell}} \\
\left(b_{j}^{\ell}, B_{j}^{\ell}\right)_{1, Q_{\ell}}
\end{array}\right] \prod_{j=1}^{2}\left[1-X_{j} t \omega^{\eta}(1-\omega)^{\mu}\right]^{-\sigma_{j}} d \omega
$$




$$
\begin{gathered}
=\Gamma(\gamma-\alpha) \sum_{n=0}^{\infty}(\gamma-\alpha)_{\mu n} g_{n}^{\sigma_{1}, \sigma_{2}}\left(X_{1}, X_{2}\right) t^{n} \\
\cdot H_{A+1, C+1:\left[P_{1}, Q_{1}\right] ; \ldots ;\left[P_{r}, Q_{r}\right]}^{0, \lambda+1:\left(M_{1}, N_{1}\right) ; \ldots ;\left(M_{r}, N_{r}\right)}\left[\begin{array}{l}
{\left[(1-\alpha-\eta n): \xi_{1}, \ldots, \xi_{r}\right]:\left[a_{j}^{\prime} ; A_{j}^{\prime}\right] ; \ldots ;\left[a_{j}^{r} ; A_{j}^{r}\right] ;} \\
{\left[(1-\gamma-\eta n-\mu n): \xi_{1}, \ldots, \xi_{r}\right]:\left[b_{j}^{\prime} ; B_{j}^{\prime}\right] ; \ldots ;\left[b_{j}^{r}, B_{j}^{r}\right] ; Z_{1}^{\xi_{1}}, \ldots, Z_{r}^{\xi_{r}}}
\end{array}\right],
\end{gathered}
$$

Also, by the application of the Theorem 2 under the conditions stated in (21) and making use of the generating relation (32),we obtain

$$
\begin{aligned}
& \int_{0}^{1} \omega^{\beta-1}(1-\omega)^{\alpha-1} \prod_{\ell=1}^{r} H_{P_{\ell}, Q_{\ell}}^{M_{\ell}, N_{\ell}}\left[\left(\omega z_{\ell}\right)^{z_{\ell}} \mid \begin{array}{c}
\left(a_{j}^{\ell}, A_{j}^{\ell}\right)_{1, P_{\ell}} \\
\left(b_{j}^{\ell}, B_{j}^{\ell}\right)_{1, Q_{\ell}}
\end{array}\right] \\
& . \prod_{j=1}^{M}\left[1-\frac{2 \omega k_{j}^{2}}{k_{j}^{2}-1}\right]^{-L_{j}} \prod_{i=1}^{N}\left[1-\frac{\left(\delta_{i}-\rho_{i}\right) \omega}{\left(1+\delta_{i}\right)}\right]^{-\lambda_{i}} \prod_{q=1}^{2}\left[1-X_{q} t \omega^{\eta}(1-\omega)^{\mu}\right]^{-\sigma_{q}} d \omega \\
& =T_{1} T_{2} \Gamma(\alpha) \sum_{n=0}^{\infty} g_{n}^{\sigma_{1}, \sigma_{2}}\left(X_{1}, X_{2}\right) t^{n}(\alpha)_{\mu n} H_{A+1, C+1:\left[P_{1}, Q_{1}\right] ; \ldots ;\left[P_{r}, Q_{r}\right]}^{0, \lambda+1:\left(M_{1}, N_{1}\right) ; \ldots ;\left(M_{r}, N_{r}\right)} \\
& .\left[\begin{array}{l}
{\left[(1-\beta-\eta n): \xi_{1}, \ldots, \xi_{r}, 1, \ldots, 1,1, \ldots, 1\right]:\left[a_{j}^{\prime} ; A_{j}^{\prime}\right] ; \ldots ;\left[a_{j}^{r} ; A_{j}^{r}\right] ;} \\
\left.\left[(1-\beta-\alpha-\eta n-\mu n): \xi_{1}, \ldots, \xi_{r}, 1, \ldots, 1,1, \ldots, 1\right]:\left[b_{j}^{\prime} ; B_{j}^{\prime}\right] ; \ldots ;\left[b_{j}^{r}, B_{j}^{r}\right] ;\left(Z_{1}\right)^{\xi_{1}}, \ldots,\left(Z_{r}\right)^{\xi_{r}}\right] .
\end{array}\right.
\end{aligned}
$$

(III) Consider the well-known generating function

$$
F(x, t)=e^{-x t}=\sum_{n=0}^{\infty} \frac{(-1)^{n} x^{n} t^{n}}{n !},
$$

and by the use of the Theorem 1, under the conditions stated with (18), we find

$$
\begin{gathered}
\int_{0}^{1} e^{-x t\left[\omega^{\eta}(1-\omega)^{\mu}\right]} \omega^{\alpha-1}(1-\omega)^{\gamma-\alpha-1} \prod_{\ell=1}^{r} H_{P_{\ell}, Q_{\ell}}^{M_{\ell}, N_{\ell}}\left[\left(\omega z_{\ell}\right)^{\xi_{\ell}} \mid \begin{array}{c}
\left(a_{j}^{\ell}, A_{j}^{\ell}\right)_{1, P_{\ell}} \\
\left(b_{j}^{\ell}, B_{j}^{\ell}\right)_{1, Q_{\ell}}
\end{array}\right] d \omega \\
=\Gamma(\gamma-\alpha) \sum_{n=0}^{\infty}(\gamma-\alpha)_{\mu n} \frac{(-1)^{n} x^{n} t^{n}}{n !} H_{\left.A+1, C+1:\left[P_{1}, Q_{1}\right] ; \ldots ; P_{r}, Q_{r}\right]}^{0, \lambda+1:\left(M_{1}, N_{1}\right) ; \ldots ;\left(M_{r}, N_{r}\right)} \\
.\left[\begin{array}{l}
{\left[(1-\sigma-\eta n): \xi_{1}, \ldots, \xi_{r}\right]:\left[a_{j}^{\prime} ; A_{j}^{\prime}\right] ; \ldots ;\left[a_{j}^{r} ; A_{j}^{r}\right] ;} \\
{\left[(1-\gamma-\eta n-\mu n): \xi_{1}, \ldots, \xi_{r}\right]:\left[b_{j}^{\prime} ; B_{j}^{\prime}\right] ; \ldots ;\left[b_{j}^{r}, B_{j}^{r}\right] ;}
\end{array}\left(Z_{1}\right)^{\xi_{1}}, \ldots,\left(Z_{r}\right)^{\xi_{r}}\right],
\end{gathered}
$$

Also, by the application of Theorem 2 with the condition surrounding (21) and on using (36), we have the following useful integral

$$
\begin{aligned}
& \int_{0}^{1} e^{-x t}\left[\omega^{\eta}(1-\omega)^{\mu}\right] \omega^{\beta-1}(1-\omega)^{\alpha-1} \prod_{\ell=1}^{r} H_{P_{\ell}, Q_{\ell}}^{M_{\ell}, N_{\ell}}\left[\left(\omega z_{\ell}\right)^{\xi_{\ell}} \mid \begin{array}{c}
\left(a_{j}^{\ell}, A_{j}^{\ell}\right)_{1, P_{\ell}} \\
\left(b_{j}^{\ell}, B_{j}^{\ell}\right)_{1, Q_{\ell}}
\end{array}\right] \\
& \quad \cdot \prod_{j=1}^{M}\left[1-\frac{2 \omega k_{j}^{2}}{k_{j}^{2}-1}\right]^{-L_{j}} \prod_{i=1}^{N}\left[1-\frac{\left(\delta_{i}-\rho_{i}\right) \omega}{\left(1+\delta_{i}\right)}\right]^{-\lambda_{i}} d \omega \\
& =T_{1} T_{2} \Gamma(\alpha) \sum_{n=0}^{\infty} \frac{(-1)^{n} x^{n} t^{n}(\alpha)_{\mu n}}{n !} H_{A+1, C+1:\left[P_{1}, Q_{1}\right] ; \ldots ;\left[P_{r}, Q_{r}\right]}^{0, \lambda+1:\left(M_{1}, N_{1}\right) ; \ldots ;\left(M_{r}, N_{r}\right)}
\end{aligned}
$$




$$
\cdot\left[\begin{array}{l}
{\left[(1-\beta-\eta n): \xi_{1}, \ldots, \xi_{r}\right]:\left[a_{j}^{\prime} ; A_{j}^{\prime}\right] ; \ldots ;\left[a_{j}^{r} ; A_{j}^{r}\right] ;} \\
{\left[(1-\beta-\alpha-\eta n-\mu n): \xi_{1}, \ldots, \xi_{r}\right]:\left[b_{j}^{\prime} ; B_{j}^{\prime}\right] ; \ldots ;\left[b_{j}^{r}, B_{j}^{r}\right] ;}
\end{array}\left(Z_{1}\right)^{\xi_{1}}, \ldots,\left(Z_{r}\right)^{\xi_{r}}\right] .
$$

It can be seen that the theorems (Theorem 1 and Theorem 2 along with and their corollaries) discussed in this article provide generalizations to a number of the known elliptic-type integrals. Furthermore, these Theorems also have their wide applications in formulation of various new elliptic-type integrals and a great use in solving a very spacious class of Euler-type integrals in terms of different generalized functions.

\section{Acknowledgements}

The authors are grateful to Professor H.M. Srivastava, (University of Victoria, Canada) for his kind help and valuable suggestions in the preparation of this paper. The authors are also thankful to the worthy referee for his valuable suggestions for the improvement of the paper.

\section{References}

[1] M. J. Berjer and J. C. Lankin, Sample calculation of gamma ray penetration into shelters. Contribution of sky shine and roof contamination, J. Res. N. B. S., 60(1958), 109-116.

[2] J. Bjorkberg and G. Kristensson, Electromagnetic scattering by a perfectly conducting elliptic disc, Canad. J. Phys., 65 (1987), 723-734.

[3] P. F. Byrd and M. D. Friedman, Handbook of elliptic integrals for Engineers and Scientists, Springer, Berlin, 1971.

[4] V. B. L. Chaurasia and S. C. Pandey, Unified elliptic-type integral and asymptotic formulas, Demonstratio Mathematica, 41(2008), 531-541.

[5] L. F. Epstein and J. H. Hubbell, Evaluation of a generalized elliptic type integral, J. Res. N.B.S., 67 (1963), 1-17.

[6] J. D. Evans, J. H. Hubbell and V. D. Evans, Exact series solution to the Epstein-Hubbell generalized elliptic-type integral using complex variable residue theory, Appl. Math. Comp., 53 (1993), 173-189.

[7] M. L. Glasser and S. L. Kalla, Recursion relations for a class of generalized elliptic-type integrals, Rev. Tec. Ing. Univ. Zulia., 12 (1989), 47-50.

[8] J. H. Hubbell, R. L. Bach and R. J. Herbold, Radiation field from a circular disk source, J. Res. N.B. S., 65 (1961), 249-264.

[9] S. L. Kalla, Results on generalized elliptic-type integrals. Mathematical Structure-Computational mathematics-mathematical Modelling, 2, 216-219, Sofia: Publ. House Bulgar. Acad. Sci., 1984.

[10] S. L. Kalla, The Hubbell rectangular source integral and its generalizations, Radiat. Phys. Chem., 41 (1993), $775-781$.

[11] S. L. Kalla, S. Conde and J. H. Hubbell, Some results on generalized elliptictype integrals, Appl. Anal., 22 (1986), 273-287.

[12] S. L. Kalla and B. Al-Saqabi, On a generalized elliptic-type integral, Rev. Bra. Fis.; 16(1986), 145-156.

[13] S. L. Kalla, C. Leubner and J. H. Hubbell, Further results on generalized Elliptic-type integrals, Appl. Anal., 25 (1987), 269-274.

[14] S. L. Kalla and V. K. Tuan, Asymptotic formulas for generalized Elliptic-type integrals, Comput. Math. Appl., 32 (1996), 49-55.

[15] E. L. Kaplan, Multiple elliptic integrals, J. Math. And Phys., 29 (1950), 69-75.

[16] S. S. Khadia and A. X. Goyal, On the generalized function ofn-variables, Vijnana Parishad Anusandhan Patrika, 13(1970), 191-201. 
[17] P. Kllinga and S. M. Khanna, Dose rate to the inner ear during Mosebauer experiments, Phys. Med. Biol., 28 (1983), 359-366.

[18] B. N. Al-Saqabi, A generalization of elliptic-type integrals, Hadronic J., 10 (1987), 331-337.

[19] A. Al-Zamel and V. K. Tuan, Kalla, S.L.Generalized Elliptic-type integrals and asymptotic formulas, Appl. Math. Comput., 114 (2000), 13-25.

[20] J. Matera, L. Galue and S. L. Kalla, Asymptotic expansions for some elliptictype integrals, Raj. Acad. Phy. Sci., $\mathbf{1}$ (2002), 71-82.

[21] A. M. Mathai and R. K. Saxena, The H-Function with Applications in Statistics and other Disciplines, New York, Halsted Press, 1978.

[22] Ch. W. Mohammed, Bilinear and bilateral generating functions of generalized Polynomials, J. Austral. Math. Soc. Ser. B., 39 (1997), 257-270.

[23] M. Salman, Generalized elliptic-type integrals and their representations, Appl. Math. Comput., 181(2006), 1249-1256.

[24] S. Saran, Theorems on bilinear generating functions, Indian J. Pure Appl. Math., 3(1972), 12-20.

[25] R. K. Saxena and S. L. Kalla, Asymptotic formulas for unified Elliptic-type integrals, Int. Tran. Spec. Funct., 15(2004), 359-368.

[26] R. K. Saxena, S. L. Kalla and J. H. Hubbell, Asymptotic expansion of a unified Elliptic-type integrals, Math. Balkanica, 15 (2001), 387-396.

[27] R. K. Saxena and S. L. Kalla, A new method for evaluating Epstein-Hubbell generalized elliptic-type integrals, Int. J. Appl. Math., 2 (2000), 732-742.

[28] R. K. Saxena and M. A. Pathan, Asymptotic formulas for unified elliptic-type integrals, Demonstratio Mathematica, 36(2003), 579-589.

[29] R. N. Siddiqi, On a class of generalized elliptic-type integrals, Rev. Barsileira Fis., 19 (1989), $137-147$.

[30] H. M. Srivastava, Infinite series of certain products involving Appell's double hypergeometric functions I, Glasnik Mat., 4(1969), 67-73.

[31] H. M. Srivastava and S. Bromberg, Some families of generalized elliptic-type integrals, Math. Comput. Modelling, 21(1995), 29-38.

[32] H. M. Srivastava, K. C. Gupta and S. P. Goyal, The H-Functions of One and Two Variables with Applications, South Asian Publishers, New Delhi, 1982.

[33] H. M. Srivastava and R. Panda, Some bilateral generating functions for a class of generalized hypergeometric polynomials, J. Reine Angew. Math., 283/284(1976), 265-274.

[34] H. M. Srivastava and H. L. Manocha, A Treatise on Generating Functions, Ellis Horwood Ltd., Chichester, 1985.

[35] H. M. Srivastava and R. N. Siddiqi, A unified presentation of certain families of elliptic-type integrals related to radiation field problems, Radiant, Phy. Chem., 46 (1995), 303-315.

[36] H. M. Srivastava and Y. N. Yeh, Certain theorems on bilinear and bilateral generating function, ANZIAM J., 43 (2002), 567-574.

Department of Mathematics, University of Rajasthan, Jaipur-302055 (Rajasthan), India.

E-mail:drvblc@yahoo.com

Department of Mathematics, Government Post-Graduate College, Neemuch-458441 (MP), India.

E-mail: meghwal66@gmail.com 